\section{L’apéline, \\ un inhibiteur \\ naturel de l'effet \\ antidiurétique de \\ la vasopressine}

Catherine Llorens-Cortès, Alain Beaudet

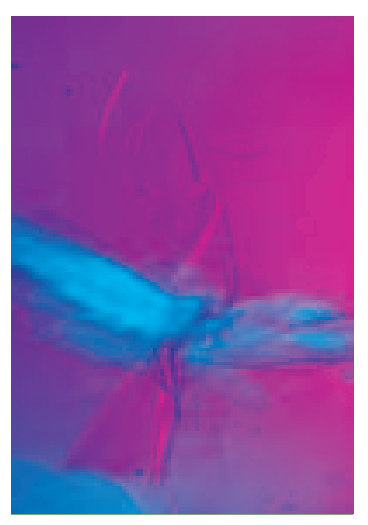

L'histoire de l'apéline commence avec le clonage de I'ADNc du récepteur APJ à partir d'une banque génomique humaine par O’Dowd et al., en 1993 [1]. Il s'agit d'un récepteur orphelin de 380 acides aminés qui appartient à la famille des récepteurs à sept domaines transmembranaires couplés aux protéines $G$ (RCPG) et qui partage $31 \%$ d'identité de séquence protéique avec le récepteur de type 1 des angiotensines (ATl), d'où son nom APJ pour protéine putative reliée au récepteur ATl. Ce récepteur a depuis été cloné chez la souris [2] puis chez le rat [3, 4]. Parallèlement, Tatemoto et al. [5] isolaient, fin 1998, le ligand endogène du récepteur APJ humain à partir d'extraits d'estomac de bœuf en utilisant une méthode de criblage fondée sur la mesure des variations de $\mathrm{pH}$ extracellulaire à l'aide d'un appareil, le Cytosenseur. Ils nommèrent le peptide isolé « apéline », pour ligand endogène pour le récepteur APJ. II s'agit d'un peptide de 36 acides aminés (apéline 36), issu d'un large précurseur, la proapéline, composé de 77 acides aminés. Le gène de l'apéline humaine est situé sur le chromosome $X$ et la phase ouverte de lecture est localisée dans deux exons ( 1 et 2 ) séparés par un intron d'en-

Article reçu le 5 janvier 2005, accepté le 8 juin 2005. 
viron $6 \mathrm{~kb}$. La partie non codante en 3' se retrouve aussi dans deux exons (2 et 3 ). Ces données peuvent expliquer la présence de deux transcrits de taille différente, de 3,0 et $3,5 \mathrm{~kb}$, en proportions variables suivant les tissus étudiés [7]. La proapéline a été isolée dans différentes espèces [5-7] et l'alignement des séquences de souris, rat, bœuf et homme a révélé une conservation stricte des 17 derniers acides aminés carboxyterminaux correspondant à l'apéline-17 ou K17F (Figure 1). In vivo, la proapéline donne naissance à différentes formes moléculaires d'apéline: dans le cerveau et le plasma de rat, on retrouve majoritairement la forme pyroglutamyl de l'apéline 13, p\&l3F (Figure 1) et en plus faible quantité, K17F [8] ; dans le testicule et l'utérus, c'est l'apéline 36 qui prédomine, alors que, dans la glande mammaire, on retrouve à la fois l'apéline 36 et pદl3F [9].

A

\section{Proapéline}

Bœuf $\quad M N L R R C V Q A L L L L W L C L S A V C G G P L L Q T S D$ Homme $\quad M N L R L C V \rho A L L L L W L S L T A V C G G S L M P L P D 30$ Rat $\quad M N L S F C V P A L L L L W L S L T A V C G V P L M L P P D \quad 30$ Souris $\quad M N L R L C \vee P A L L L L W L S L T A \vee C G V P L M L P P D \quad 30$ Bœuf $\quad G K E M E \varepsilon G T / R$ Y L V P PRGPRSGPGPW $P G G R R 60$ Homme GNGLEDGNVRHLVPPRGSRNGPGPW $P$ G GR R 60 Rat $\quad G K G L E \& G N M R Y L V K P R T S R T G P G A W \rho G G R R 60$ Souris $\quad G T G L E \varepsilon G S M R Y L V K P R T S R T G P G A W Q G G R R 60$

$\begin{array}{llll}\text { Bœuf } & \text { K F R R P R P R L S H K G P M P F } 77\end{array}$

Homme K F R R P R P R L S H K G P M P F 77

Rat $\quad$ K F R R P R P R S H K G P M P F 77

\begin{tabular}{lllll} 
Souris & K F R R P R P R L H K G P M P F & $\mathbf{7 7}$ \\
\hline
\end{tabular}

apéline 17 ou K17F

B

回 $\mathrm{K} 17 \mathrm{~F}$

$\triangle \mathrm{p} \varepsilon 13 \mathrm{~F}$

Lys-Phe-Arg -Arg -Gln -Arg - Pro-Arg - Leu-Ser-His - Lys-Gly - Pro-Met-Pro Phe pGlu -Arg -Pro-Arg -Leu-Ser-His -Lys-Gly-Pro-Met-ProPhe Fragments d'apéline actifs

$\boldsymbol{\nabla}$ R10F

- G5F

Arg-Leu-Ser-His -Lys-Gly-Pro-Met-ProPhe Gly-Pro-Met-Pro Phe $]$ Fragments d'apéline inactifs

C

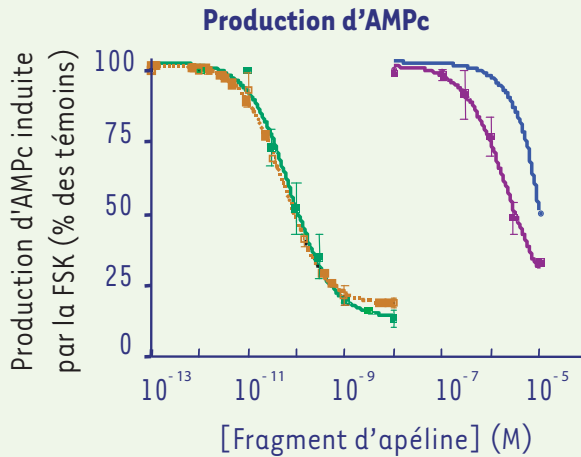

D

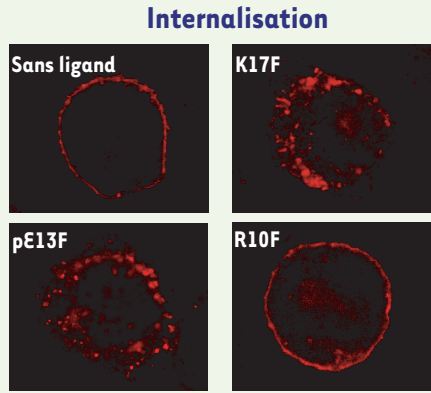

Figure 1. A. Séquence primaire du précurseur de l'apéline, la proapéline, chez l'homme, le bœuf, le rat et la souris. La proapéline a été isolée dans différentes espèces [5-7] et l'alignement des séquences de souris, rat, bœuf et homme a révélé une conservation stricte des 17 derniers acides aminés carboxyterminaux correspondant à l'apéline-17 ou K17F. B. Structure primaire de différents fragments d'apéline, apéline-17 (K17F), forme pyroglutamyl de l'apéline-13 (pE13F), apéline-10 (R10F), apéline-5 (G5F). In vivo, la proapéline donne naissance à différentes formes moléculaires d'apéline: dans le cerveau et le plasma de rat, on retrouve majoritairement la forme pyroglutamyl de l'apéline 13, pE13F et en plus faible quantité, K17F [8]. Ces deux peptides présentent une forte affinité (nM) pour le récepteur APJ, alors qu'elle est très faible pour Rl0F et G5F. C. Inhibition de la production d'AMPc induite par la forskoline (FSK) par différents fragments d'apéline. Les fragments sont appliqués sur des cellules CHO exprimant de façon stable le récepteur de l'apéline étiqueté à son extrémité carboxyterminale avec une protéine fluorescente l'£GFP (R-Apéline-EGFP) [12, 13] : K17F (courbe d'inhibition en orange), pEl3F (en vert), R10F (en violet), G5F (en bleu). D. Internalisation du récepteur de l'apéline (R-Apéline-EGFP) dans une lignée de cellules CHO exprimant de façon stable ce récepteur sous l'action de différents fragments d'apéline (K17F, p\&13F, R10F) [12, 13]. 
À l'aide de K17F et d'autres fragments d'apéline tronqués à l'extrémité aminoterminale de K17F, plusieurs laboratoires ont montré que les récepteurs murin et humain de l'apéline, exprimés de façon stable dans des cellules ovariennes de hamster chinois ( $\mathrm{CHO}$ ), sont couplés négativement à l'adénylate cyclase. Les fragments les plus actifs du propeptide sont K17F ainsi que l'apéline 13 (Q13F) et pEl3F [4, 6, 10, 11] (Figure 1). Ces mêmes fragments induisent l'internalisation du récepteur de l'apéline [11-13]. De plus, la stimulation du récepteur de l'apéline par pદl3F entraîne l'activation de la voie des MAP-kinases et des PI3-kinases [14].

\section{Distribution de l'apéline et de son récepteur dans le cerveau}

Le développement d'un anticorps polyclonal de haute affinité et sélectivité contre $\mathrm{KITF}[8,12]$ a permis de visualiser, pour la première fois chez le rat, la présence de neurones apélinergiques dans le système nerveux central. Les corps cellulaires de ces neurones sont particulièrement abondants dans les structures de l'hypothalamus et $\mathrm{du}$ bulbe rachidien impliquées dans le contrôle neuroendocrinien, le comportement dipsique et la régulation de la pression artérielle, notamment dans la partie magnocellulaire des noyaux paraventriculaire (PVN) et supra-optique (SON) de l'hypothalamus, le noyau arqué, le noyau réticulé latéral et le noyau ambigu [15]. Une forte densité de fibres et de terminaisons apélinergiques est également présente dans la couche interne de l'éminence médiane et dans l'hypophyse postérieure [12, 16], suggérant que les neurones apélinergiques du PVN et du SON se projettent, comme les neurones magnocellulaires vasopressinergiques et ocytocinergiques, dans I'hypophyse postérieure. Des études de double marquage en immunofluorescence ont confirmé cette hypothèse en montrant que l'apéline était en fait localisée dans les mêmes neurones magnocellulaires hypothalamiques que la vasopressine (AVP) [8, 17] et l'ocytocine [16]. De nombreuses fibres apélinergiques ont également été visualisées dans la région située le long du troisième ventricule, la lame terminale (composé de l'organe subfornical [SFO], l'organe vasculaire de la lame terminale [OVLT] et le noyau médian préoptique [MnPO]), impliquée dans la régulation de la prise de boisson [18].

Tout comme l'apéline, le récepteur de ce peptide est très largement distribué dans le système nerveux central du rat $[3,4,7]$. Ainsi, on observe, par hybridation in situ, l'expression de l'ARNm du récepteur de l'apéline dans le cortex cérébral, l'hippocampe et des structures contenant les neurones mono-aminergiques (substance noire, noyau raphé dorsal, locus cœruleus). Une expression très forte est détectée dans les noyaux hypothala- miques riches en apéline, dont le PVN, le SON et le noyau arqué, ainsi que dans la glande pinéale et les lobes antérieur et intermédiaire de l'hypophyse. De plus, des études en double marquage associant l'immunocytochimie et l'hybridation in situ, ont démontré que dans le PVN et le SON, les récepteurs de l'apéline $[12,19]$, tout comme ceux de l'AVP de type la et lb (Vla et Vlb) [20], sont synthétisés par les neurones magnocellulaires vasopressinergiques. On sait depuis longtemps que ces neurones neurosécrétoires libèrent I'AVP, un peptide vasoconstricteur et anti-aquarétique, dans les capillaires fenêtrés de la neurohypohyse, en réponse aux variations d'osmolalité plasmatique et de volémie [21, 22] ou sous l'action de différentes neurohormones comme l'angiotensine II/III et les peptides natriurétiques [23, 24]. On ignorait cependant jusqu'à tout récemment quelle était la réponse apélinergique à ces mêmes stimulus.

\section{Implication de l'apéline dans la régulation de l'équilibre hydrique et des fonctions cardiovasculaires}

La colocalisation AVP/apéline dans les neurones magnocellulaires de l'hypothalamus et l'association étroite des récepteurs des deux peptides avec ces mêmes neurones nous a conduits à postuler qu'indépendamment du rétrocontrôle exercé par l'AVP sur sa propre libération, l'apéline pouvait réguler la libération de l'AVP. Cette hypothèse a été testée dans le modèle de la rate en lactation, dans lequel on retrouve une hyperactivité des neurones magnocellulaires vasopressinergiques [25] destinée à conserver au mieux le contenu hydrique de l'organisme pour une production optimale de lait. Dans ce modèle, l'apéline (K17F) injectée par voie centrale diminue l'activité électrique phasique des neurones vasopressinergiques, réduit la sécrétion d'AVP dans la circulation sanguine et entraîne une diurèse aqueuse [8] (Figure 2). De la même façon, une réduction importante de la sécrétion systémique d'AVP est observée après injection intracérébroventriculaire des fragments d'apéline K17F ou pEl3F chez des souris déshydratées pendant 24 heures [12], condition qui entraîne elle aussi une hyperactivité des neurones vasopressinergiques. On peut conclure de ces résultats que l'apéline pourrait, comme l'AVP, être libérée au niveau somatodendritique et exercer un effet inhibiteur direct sur l'activité phasique des neurones vasopressinergiques et la libération systémique d'AVP via des autorécepteurs synthétisés par ces neurones. Par ce mécanisme, l'apéline jouerait donc le rôle d'un inhibiteur naturel de l'effet antidiurétique de l'AVP.

Bien que les deux peptides soient localisés dans les mêmes neurones, chez le rat déshydraté pendant 24 heures, l'hyperactivité des neurones vasopressinergiques et l'augmentation concomitante de la sécrétion systémique d'AVP s'accompagnent d'une diminution des taux d'apéline dans le plasma [8]. On observe en parallèle, par immunohistochimie quantitative, une déplétion du contenu en AVP et une augmentation du contenu en apéline des neurones vasopressinergiques du PVN et du SON [17]. L'augmentation des concentrations intracellulaires d'apéline observée chez les animaux déshydratés est inhibée par l'administration centrale d'un antagoniste sélectif des récepteurs Vl de I'AVP et est mimée par une administration intracérébroventriculaire 
d'AVP [17], suggérant qu'elle est causée par l'augmentation de libération somatodendritique d'AVP secondaire à la déshydratation. Cette interprétation implique que l'apéline et l'AVP sont libérées de façon différentielle par les neurones magnocellulaires vasopressinergiques dans lesquels elles sont colocalisées. En accord avec cette hypothèse, des études de double marquage en microscopie confocale ont montré que les deux peptides se retrouvent en grande partie dans des granules neurosécrétoires, de taille et de distribution distinctes, dans les neurones magnocellulaires [8, 17] (Figure 2).

Ces résultats démontrent que lors d'une déshydratation, l'apéline et I'AVP jouent un rôle complémentaire. Alors que l'augmentation de libération somatodentritique de l'AVP optimise l'activité phasique des neurones vasopressinergiques $[26,27]$, facilitant la sécrétion d'AVP dans la circulation sanguine, l'apéline s'accumule dans ces neurones au lieu d'être libérée dans la circulation sanguine, provoquant une diminution de son activité inhibitrice et, par ce biais, une amplification de l'activité phasique des neurones vasopressinergiques. Cette régulation croisée de l'apéline et de l'AVP semble avoir une finalité biologique puisqu'elle permet de maintenir l'équilibre hydrique de l'organisme, en évitant une perte d'eau supplémentaire par les reins. Cette régulation fine et bien orchestrée est nécessaire puisque, de façon intrinsèque, I'AVP et l'apéline ont des actions opposées sur la diurèse.

II est possible qu'en plus de cet effet central, l'apéline joue un rôle direct sur la diurèse à la périphérie. En effet, l'expression de l'ARNm du récepteur de l'apéline a été detectée dans le rein chez le rat $[3,28]$ et une immunoréactivité apéline a été visualisée dans le canal collecteur chez l'homme [29]. Enfin, en accord avec un rôle de l'apéline dans la régulation de l'équilibre hydrique qui dépend non seulement de la sécrétion d'AVP, mais aussi de la régulation de la soif et de la prise de sel [30], des fibres et des terminaisons apélinergiques

L'apéline 17 ou K17F (๑) injectée par voie centrale, en agissant sur ses récepteurs localisés sur les neurones AVP :

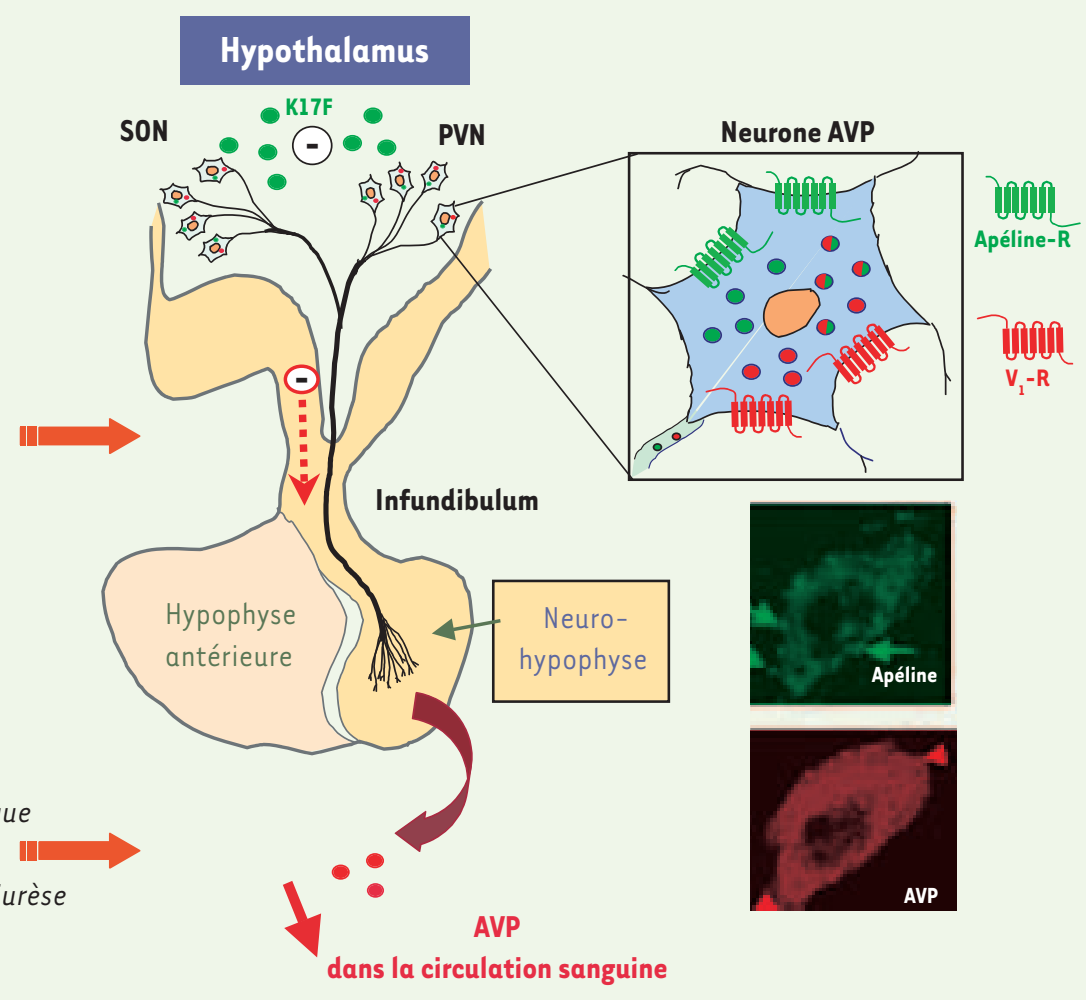

(2) inhibe la sécrétion systémique d'AVP (○), ce qui a pour conséquence d'augmenter la diurèse
(1) inhibe l'activité électrique phasique des neurones AVP 
ont été visualisées dans les centres de la soif (SFO, OVLT, MnPO) [15] et l'injection d'apéline par voie intracérébroventriculaire chez le rat déshydraté pendant $24 \mathrm{~h}$ réduit la quantité d'eau ingérée [12].

Outre ces effets sur le contrôle de l'équilibre hydrique, l'apéline a aussi des effets cardiovasculaires propres. L'ARNm des récepteurs de l'apéline a été détecté dans le myocarde et l'endothélium vasculaire [3]. L'apéline injectée par voie systémique diminue la pression artérielle $[7,12,31]$ via un mécanisme dépendant de la production de NO [31]. Dans le cœur du rongeur, l'apéline augmente la force contractile du myocarde par un effet inotrope positif tout en diminuant les pré- et post-charges [32-35]. Une augmentation de l'immunoréactivité apéline a été observée dans le plasma de patients ayant une insuffisance cardiaque à un stade précoce, alors qu'une diminution apparaîtrait à un stade plus sévère [36]. Ces données suggèrent que le récepteur de l'apéline de par les effets inotrope positif, vasodilatateur et diurétique de son ligand, pourrait représenter une cible thérapeutique potentielle dans le traitement de l'insuffisance cardiaque.

\section{Conclusions}

La découverte de l'apéline comme ligand endogène du récepteur orphelin APJ, constitue une avancée importante au plan fondamental et, potentiellement, au plan clinique. Au plan fondamental, elle représente un bon exemple de la validité de l'approche de «désorphanisation » des récepteurs orphelins pour l'identification de nouveaux peptides bio-actifs et de nouvelles cibles thérapeutiques. Les données expérimentales obtenues jusqu'ici démontrent que l'apéline, par son rôle inhibiteur sur l'activité électrique phasique des neurones vasopressinergiques et la sécrétion systémique

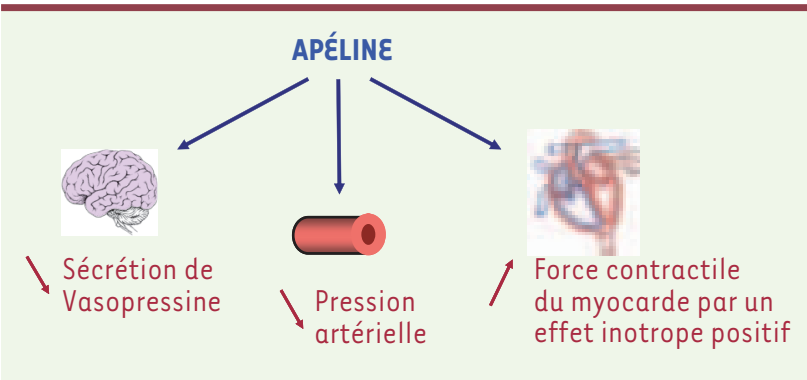

Figure 3. Récapitulatif des actions biologiques de l'apéline au niveau central et périphérique. L'apéline, un intégrateur de plusieurs fonctions impliquées dans le contrôle de l'équilibre hydrique et des fontions cardiovasculaires, diminue la sécrétion de vasopressine et la pression artérielle et augmente la force contractile du myocarde $[7$, $8,12,32,34]$. de vasopressine, induit une diurèse. À la périphérie, elle diminue la pression artérielle, augmente la force contractile du myocarde tout en diminuant les pré- et post-charges, des actions opposées à celles de la vasopressine et de l'angiotensine II. L'ensemble de ces données (Figure 3) montre que ce nouveau (neuro)peptide vaso-actif circulant pourrait jouer un rôle crucial dans le maintien de l'équilibre hydrique et des fonctions cardiovasculaires. Sur le plan clinique, le développement d'analogues non peptidiques du récepteur de l'apéline, fondé sur la connaissance de la structure de l'apéline et de celle de son récepteur, pourrait offrir de nouveaux outils thérapeutiques pour le traitement des sécrétions inappropriées d'AVP, de l'insuffisance cardiaque et de l'insuffisance rénale. $\diamond$

\section{REMERCIEMENTS}

Une grande partie des travaux présentés dans cette revue ont été réalisés en collaboration avec les unités de neuroendocrinologie Inserm U.413 ( Pr H. Vaudry), Inserm U.308 (Dr A. Burlet) et CNRS UMR 5101 (Dr F.Moos) ainsi qu'avec le laboratoire de neuromorphologie du Pr M. Palkovits (Université Semmelweis, Budapest, Hongrie). Ces travaux ont été financés par l'Inserm, le CNRS, le Fonds de la Recherche en Santé du Québec, les Instituts de recherche en santé du Canada, le programme de coopération France-Hongrie BALATON et la Société Française d'Hypertension.

\section{SUMMARY}

Apelin, a neuropeptide that counteracts vasopressin secretion

Apelin is a peptide that was recently isolated as the endogenous ligand for the human orphan APJ receptor, a $G$ protein-coupled receptor which shares $31 \%$ amino-acid sequence identity with the angiotensin type 1 receptor. Apelin naturally occurs in the brain and plasma as 13 (pE13F) and 17 amino-acid (K17F) fragments of a single pro-peptide precursor. In transfected $\mathrm{CHO}$ cells, K17F and pEl3F bind with high affinity to the rat APJ receptor, promote receptor internalization, and inhibit forskolin-induced cAMP formation. In the same cells, pEl3F activates MAP kinase and PI3 kinase pathways. Apelin and APJ receptors are both widely distributed in the brain but are particularly highly expressed in the supraoptic (SON) and paraventricular (PVN) hypothalamic nuclei. Dual labeling studies demonstrate that within these two nuclei, apelin and its receptor are colocalized with vasopressin (AVP) in a subset of magnocellular neurons. In lactating rats, characterized by increases in both synthesis and release of AVP, central injection of apelin inhibits the phasic electrical activity of AVP neurons, reduces plasma AVP levels, and increases aqueous diuresis. Moreover, water deprivation, while increasing the activity of AVP neurons, reduces plasma apelin concentrations and induces an intra-neuronal pile up of the peptide, thereby decreasing the inhibitory effect of apelin on AVP release and preventing additional water loss at the kidney level. Taken together, these data demonstrate that apelin counteracts the effects of AVP in the maintenance of body fluid homeostasis. In addition, apelin and its receptor are present in the cardiovascular system, i.e. heart, kidney and vessels. Systemically administered apelin reduces arterial blood pressure, increases cardiac contractility 
and reduces cardiac loading. The development of non peptidic analogs of apelin may therefore offer new therapeutic avenues for the treatment of cardiovascular disorders. $\Delta$

\section{RÉFÉRENCES}

1. O'Dowd BF, Heiber M, Chan A, et al. A human gene that shows identity with the gene encoding the angiotensin receptor is located on chromosome 11. Gene 1993; 136 : $355-60$

2. Devic $\varepsilon$, Rizzoti $K$, Bodin $S$, et al. Amino acid sequence and embryonic expression of msr/apj, the mouse homolog of Xenopus X-msr and human APJ. Mech Dev $1999 ; 84$ : 199-203.

3. O'Carroll A, Selby TL, Palkovits M, et al. Distribution of mRNA encoding B78/apj, the rat homologue of the human APJ receptor, and its endogenous ligand apelin in brain and peripheral tissues. Biochim Biophys Acta 2000 ; 1492 : 72-80.

4. De Mota N, Lenkei Z, Llorens-Cortes C. Cloning, pharmacological characterization and brain distribution of the rat apelin receptor. Neuroendocrinology $2000 ; 72$ : 400-7.

5. Tatemoto K, Hosoya M, Habata Y, et al. Isolation and characterization of a novel endogenous peptide ligand for the human APJ receptor. Biochem Biophys Res Commun $1998 ; 251: 471-6$

6. Habata Y, Fujii R, Hosoya M, et al. Apelin, the natural ligand of the orphan receptor APJ, is abundantly secreted in the colostrum. Biochim Biophys Acta $1999 ; 13: 25$ 35.

7. Lee DK, Cheng R, Nguyen T, et al. Characterization of apelin, the ligand for the APJ receptor. J Neurochem $2000 ; 74: 34-41$.

8. De Mota N, Reaux-Le Goazigo A, દl Messari S, et al. Apelin, a potent diuretic neuropeptide counteracting vasopressin actions through inhibition of vasopressin neuron activity and vasopressin release. Proc Natl Acad Sci USA 2004 ; 101 : 10464 9.

9. Kawamata Y, Habata Y, Fukusumi S, et al. Molecular properties of apelin : tissue distribution and receptor binding. Biochim Biophys Acta 2001 ; 1538: 162-71.

10. Zou MX, Liu HY, Haraguchi Y, et al. Apelin peptides block the entry of human immunodeficiency virus (HIV). FEBS Lett 2000 ; 473 : 15-8.

11. Medhurst AD, Jennings CA, Robbins MJ, et al. Pharmacological and immunohistochemical characterization of the APJ receptor and its endogenous ligand apelin. J Neurochem $2003 ; 84: 1162-72$.

12. Reaux A, De Mota N, Skultetyova I, et al. Physiological role of a novel neuropeptide, apelin, and its receptor in the rat brain. J Neurochem $2001 ; 77: 1085-96$.

13. El Messari S, Iturrioz X, Fassot C, et al. Functional dissociation of apelin receptor signaling and endocytosis : implications for the effects of apelin on arterial blood pressure. J Neurochem 2004 ; 90 : 1290-301.

14. Masri B, Morin N, Cornu M, et al. Apelin (65-77) activates p70 S6 kinase and is mitogenic for umbilical endothelial cells. FASEB J $2004 ; 18$ : 1909-11.

15. Reaux A, Gallatz K, Palkovits M, Llorens-Cortes C. Distribution of apelinsynthesizing neurons in the adult rat brain. Neuroscience $2002 ; 113: 653-62$.

16. Brailoiu, GC, Dun SL, Yang J, et al. Apelin-immunoreactivity in the rat hypothalamus and pituitary. Neurosci Lett $2002 ; 327: 193-7$.

17. Reaux-Le Goazigo A, Morinville A, Burlet A, et al. Dehydration-induced cross regulation of apelin and vasopressin immunoreactivity levels in magnocellular hypothalamic neurons. Endocrinology 2004 ; 145 : 4392-400.
18. Johnson AK, Cunningham JT, Thunhorst RL. Integrative role of the lamina terminalis in the regulation of cardiovascular and body fluid homeostasis. Clin Exp Pharmacol Physiol 1996; 23 : 183-91.

19. O'Carroll AM, Lolait SJ. Regulation of rat APJ receptor messenger ribonucleic acid expression in magnocellular neurones of the paraventricular and supraoptic nuclei by osmotic stimuli.J Neuroendocrinol $2003 ; 15: 661-6$.

20. Hurbin A, Boissin-Agasse L, Orcel H, et al. The Vla and Vlb, but not V2, vasopressin receptor genes are expressed in the supraoptic nucleus of the rat hypothalamus, and the transcripts are essentially colocalized in the vasopressinergic magnocellular neurons. Endocrinology 1998 ; 139 : 4701-7.

21. Brownstein MJ, Russell JT, Gainer H. Synthesis, transport, and release of posterior pituitary hormones. Science $1980 ; 207: 373-8$.

22. Manning M, Lowbridge J, Haldar J, et al. Design of neurohypophyseal peptides that exhibit selective agonistic and antagonistic properties. Fed Proc 1977 ; 36 : 184852.

23. Zini S, Fournie-Zaluski MC, Chauvel $\varepsilon$, et al. Identification of metabolic pathways of brain angiotensin II and III using specific aminopeptidase inhibitors : predominant role of angiotensin III in the control of vasopressin release. Proc Natl Acad Sci USA $1996 ; 93: 11968-73$.

24. Gutkowska J, Antunes-Rodrigues J, McCann SM. Atrial natriuretic peptide in brain and pituitary gland. Physiol Rev $1997 ; 77: 465-515$.

25. Poulain DA, Wakerley JB, Dyball RE. Electrophysiological differentiation of oxytocin- and vasopressin-secreting neurones. Proc R Soc Lond B Biol Sci 1977 ; $196: 367-84$.

26. Gouzenes L, Desarmenien MG, Hussy N, et al. Vasopressin regularizes the phasic firing pattern of rat hypothalamic magnocellular vasopressin neurons. J Neurosci $1998 ; 18: 1879-85$.

27. Ludwig M. Dendritic release of vasopressin and oxytocin. J Neuroendocrinol 1998 ; $10: 881-95$.

28. Hosoya M, Kawamata Y, Fukusumi S et al. Molecular and functional characteristics of APJ : tissue distribution of mRNA and interaction with endogenous ligand, apelin. J Biol Chem $2000 ; 275: 21061-7$.

29. De Falco M, De Luca L, Onori N, et al. Apelin expression in normal human tissues. In Vivo $2002 ; 16: 333-6$

30. Denton DA, McKinley MJ, Weisinger RS. Hypothalamic integration of body fluid regulation. Proc Natl Acad Sci USA 1996 ; 93 : 7397-404.

31. Tatemoto K, Takayama K, Zou MX, et al. The novel peptide apelin lowers blood pressure via a nitric oxide-dependent mechanism. Regul Pept 2001; 99: 87-92.

32. Szokodi I, Tavi P, Foldes G, et al. Apelin, the novel endogenous ligand of the orphan receptor APJ, regulates cardiac contractility. Circ Res $2002 ; 91: 434-40$.

33. Chen MM, Ashley $\varepsilon A$, Deng DX, et al. Novel role for the potent endogenous inotrope apelin in human cardiac dysfunction. Circulation $2003 ; 108: 1432-9$.

34. Berry MF, Pirolli TJ, Jayasankar V, et al. Apelin has in vivo inotropic effects on normal and failing hearts. Circulation $2004 ; 110$ : 11187-93.

35. Ashley $\varepsilon A$, Powers J, Chen $M$, et al. The endogenous peptide apelin potently improves cardiac contractility and reduces cardiac loading in vivo. Cardiovascular Res $2005 ; 65: 73-82$.

36. Földes G, Horkay F, Szokodi I, et al. Circulating and cardiac levels of apelin, the novel ligand of the orphan receptor APJ, in patients with heart failure. Biochem Biophys Res Commun 2003 ; 308 : 480-5.

\section{TIRÉS À PART}

C. Llorens-Cortès

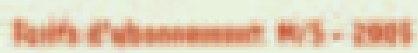
Abonnez-vous à Médecine/Sciences
$>1985-2005$, depuis 20 ans, grâce d̀ $\mathrm{m} / \mathrm{s}$, vous vivez en direct les progrès des sciences biologiques et médicales

Halletin e'cobeneenent pege 678 enat ar andre do mis
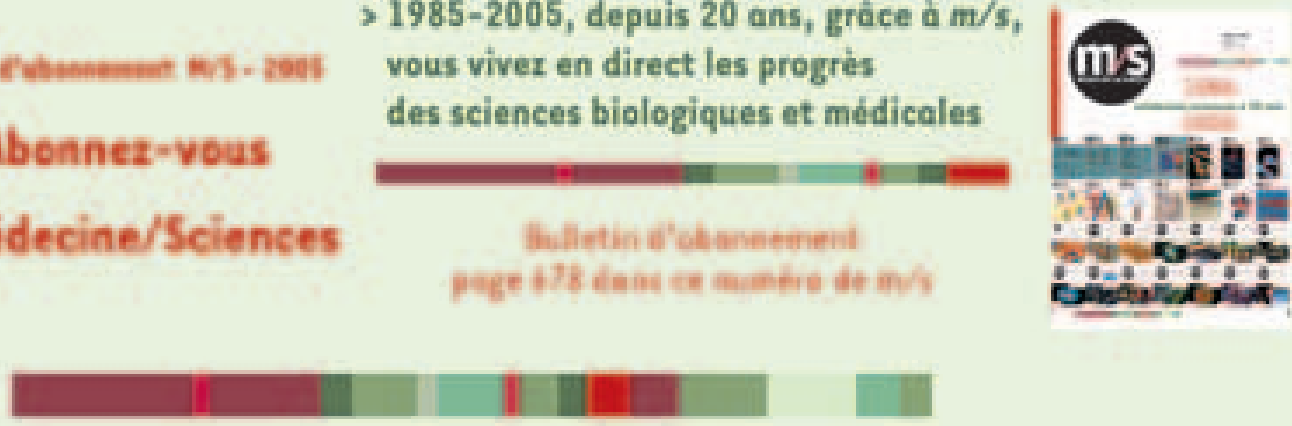